\title{
Dépenses d'énergie, composition corporelle et activité physique chez l'homme
}

\section{Luc Tappy Éliane Guenat}

L. Tappy, E. Guenat: Institut de physiologie,
Faculté de médecine, 7 , rue du Bugnon, Faculté de médecine,
1005 Lausanne, Suisse.

apports et les dépenses d'énergie. Une augmentation des dépenses énergétiques pourrait prévenir l'obésité. Elles peuvent être subdivisées en trois composantes principales. Le métabolisme de repos $(60 \%$ à $70 \%$ des dépenses totales) correspond au coût énergétique de «maintien» de l'organisme. II est peu modifié par la nutrition. La thermogenèse alimentaire $(10 \%$ des dépenses totales) correspond au coût de stockage des aliments; elle comprend aussi une composante facultative dépendant du système nerveux sympathique. Enfin, les dépenses énergétiques liées à I'activité physique représentent environ $20 \%$ des dépenses totales chez l'individu sédentaire. En l'état actuel des connaissances, une augmentation de l'activité physique est le seul moyen susceptible d'augmenter les dépenses énergétiques de I'homme. La définition du contrôle des dépenses énergétiques par le système nerveux et des mécanismes cellulaires réglant l'efficacité énergétique pourrait cependant offrir de nouvelles perspectives thérapeutiques.

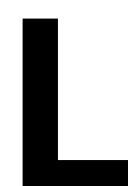

'homme dépend, pour sa survie et le maintien de ses fonctions, d'un apport continu d'énergie. Cette énergie lui est fournie par la dégradation $d$ 'adénosine triphosphate (ATP) en adénosine-diphosphate (ADP). Cette réaction permet la libération de l'énergie chimique contenue dans la molécule d'ATP, énergie qui peut être utilisée pour fournir un travail, qu'il soit électrochimique pour le maintien des gradients ioniques transmembranaires par exemple, ou mécanique lors d'une activité musculaire. La régénération d'ADP en ATP s'effectue au cours de la phosphorylation oxydative dans les mitochondries. L'énergie nécessaire à ce processus est dérivée de l'oxydation de substrats énergétiques, glucides, lipides et protides. Le rendement énergétique de la synthèse d'ATP est estimé à $60 \%$. En d'autres termes, $40 \%$ de l'énergie contenue dans les aliments sont dissipés sous forme de chaleur au cours de leur oxydation. Ces substrats sont présents en quantité variable dans l'organisme. L'organisme d'un homme de $70 \mathrm{~kg}$ contient environ 300 à $500 \mathrm{~g}$ d'hydrates de carbone sous forme de glycogène hépatique et musculaire, $6 \mathrm{~kg}$ de protéines consti- tuant le parenchyme des organes et le muscle ainsi que 7 à $15 \mathrm{~kg}$ de lipides sous forme de tissu adipeux souscutané et viscéral. Cependant, pour permettre l'oxydation continue de substrats énergétiques sans modifier la composition corporelle et principalement sans diminuer la masse protéique indispensable à la fonction des cellules de l'organisme, I'homme dépend pour sa survie d'un apport toujours renouvelé de substrats énergétiques sous forme d'aliments.

L'énergie produite est utilisée, en proportions variables, dans chaque cellule de l'organisme. Le système cardiovasculaire consomme continuellement de l'énergie pour effectuer son travail de pompage et maintenir un tonus vasculaire. Le système respiratoire consomme de l'énergie pour assurer le travail des muscles respiratoires. Enfin, les systèmes digestif et rénal consomment de l'énergie pour le transport actif de certains composés. De plus, toutes les cellules de ces systèmes sont en constant renouvellement, un processus qui implique également une consommation d'énergie [1]. Une part importante de l'énergie dépensée sert donc aux systèmes de " maintien» de l'organisme. La fonction de ces systèmes est cependant de 
permettre l'autonomie de l'individu, $c^{\prime}$ est-à-dire sa pensée et sa libre motilité. Dans cette perspective, il est donc possible [2] que l'ensemble des dépenses énergétiques soit destiné à permettre I'activité des fonctions nerveuses supérieures et l'activité physique.

\section{Mesure de la dépense d'énergie}

Les dépenses énergétiques de I'homme peuvent être évaluées par la technique de la calorimétrie indirecte. Cette technique repose sur le principe que l'oxydation des substrats énergétiques peut être évaluée par la mesure de la consommation d'oxygène et la production de $\mathrm{CO}_{2}$ de l'individu entier et que cette oxydation est pour sa quasi-totalité couplée à une synthèse $d^{\prime}$ ATP au cours du processus de la phosphorylation oxydative. De manière à évaluer la proportion de glucides, lipides et protéines oxydés, cette technique requiert également la mesure de l'excrétion urinaire d'azote [2]. Cette technique peut être appliquée aisément à l'étude du métabolisme de I'homme en utilisant un boîtier ventilé avec de l'air ambiant sur la tête de manière à collecter en continu les gaz expirés. Il est ainsi possible d'évaluer quantitativement les dépenses énergétiques globales dans différentes conditions physiologiques. Les dépenses énergétiques de 24 heures d'un individu peuvent être mesurées par la technique de la calorimétrie indirecte en chambre respiratoire. II s'agit d'une enceinte étanche, dans laquelle le sujet peut vivre pendant des périodes de 24 heures ou plus, qui permet le recueil continuel des gaz expirés. Les mesures pratiquées nécessitent évidemment le confinement dans un espace étroit, mais il est possible d'effectuer une activité physique standardisée sur tapis roulant ou sur une bicyclette ergométrique. Les dépenses énergétiques totales de l'individu peuvent aussi être estimées sur des périodes moyennes de 7 jours ou plus grâce à la méthode dite de l'eau doublement marquée [3]. Cette méthode consiste à administrer une dose d'eau marquée avec du deutérium et de I'oxygène 18. L'enrichissement en deutérium de l'eau corporelle dimi- nue progressivement et reflète les pertes d'eau de l'organisme (urines, sudation, pertes fécales et respiratoires). L'oxygène 18 quitte I'organisme non seulement par les pertes d'eau, mais également avec les émissions de $\mathrm{CO}_{2}$. En effet, il existe une équilibration entre l'eau et le $\mathrm{CO}_{2}$, catalysée par l'enzyme anhydrase carbonique, au cours de laquelle I'oxygène 18 présent dans l'eau peut être transféré sur le $\mathrm{CO}_{2}$. II s'ensuit que la décroissance de l'enrichissement en oxygène 18 de l'eau est plus rapide que celle en deutérium et peut être utilisée pour calculer la production moyenne de $\mathrm{CO}_{2}$ de l'organisme sur la période considérée. II est ensuite possible de calculer la consommation $d^{\prime}$ oxygène correspondante et, par conséquent, les dépenses énergétiques totales.

\section{Composantes}

\section{de la dépense d'énergie}

Chez le sujet étendu sur un lit, au repos, après une nuit de jeûne, les mesures effectuées permettent d'évaluer le métabolisme de base. Ce métabolisme de base correspond à I'énergie dépensée pour les fonctions de maintien de l'organisme: le travail cardiaque et respiratoire, l'énergie dépensée pour le maintien des gradients ioniques transmembranaires et I'activité du système nerveux ainsi que le turnover des constituants tissulaires représentent la part la plus importante de ces dépenses de maintien.

Les mesures du métabolisme de base montrent une très grande variabilité inter-individuelle. Cette variabilité est principalement attribuée à des différences de composition corporelle. Le métabolisme de base croît proportionnellement à la masse corporelle. La relation qui existe entre masse corporelle et métabolisme de repos diffère cependant entre l'homme et la femme et entre l'individu sédentaire et I'athlète [4]. Ces différences peuvent $s^{\prime}$ expliquer par les proportions variables de masse maigre et de masse grasse qui existent au sein de ces groupes d'individus. En effet, la masse corporelle représente un amalgame d'organes et de tissus dont le métabolisme diffère considérablement [5]. Le tissu adipeux en particulier contribue de manière variable à la masse corporelle totale et est caractérisé par des dépenses énergétiques relativement faibles. Au contraire, la masse dite "non grasse» ou «maigre» de l'organisme correspond aux parenchymes des organes et aux muscles et est caractérisée par des dépenses énergétiques nettement plus élevées. Lorsque le métabolisme de base est exprimé en fonction de la masse grasse plutôt que de la masse corporelle totale, la variabilité interindividuelle est réduite dans une proportion importante et les différences entre hommes, femmes et athlètes s'estompent [4]. Outre la composition corporelle, le métabolisme de base est réglé par des influences neurohormonales. L'effet des hormones thyroïdiennes est bien connu. Une hypothyroïdie s'accompagne d'une baisse marquée du métabolisme de base alors qu'au contraire une élévation des hormones thyroïdiennes est accompagnée d'une élévation du métabolisme de base [6]. De même, I'administration des catécholamines, ou un stress mental, augmente de manière aiguë le métabolisme de base [7]. Les mécanismes responsables de ces effets hormonaux sur le métabolisme restent incomplètement élucidés. Il est vraisemblable que I'activation de cycles métaboliques dits "futiles», au cours desquels de I'ATP est dépensé, soit impliquée. Le rôle du système nerveux sympathique est plus discuté. Chez le rongeur, une portion du métabolisme de repos peut correspondre à un découplage de la phosphorylation oxydative dans le tissu adipeux brun. Ce découplage s'opère par le biais d'une protéine spécifique, I'UCP1 (uncoupling protein 1), présente uniquement dans le tissu adipeux brun et qui, lorsqu'elle est activée, permet la dissipation de I'énergie issue de l'oxydation des substrats en chaleur en facilitant le transfert de protéines au travers de la membrane mitochondriale. L'activité de I'UCP1 chez le rongeur est augmentée par I'activité du système nerveux sympathique [8]. Chez I'homme, il n'existe pas de tissu adipeux brun en quantités appréciables. D'autres types de protéines dont la séquence laisse supposer des propriétés de découplage de la phosphorylation oxydative ont récemment été décrits: UCP2 dans de nombreux organes $\left(m / s\right.$ 1997, $\left.n^{\circ} 4, p .607\right)$, 
UCP3 dans le muscle squelettique. Un rôle de ces protéines découplantes dans le contrôle du métabolisme de base n'a cependant pas été démontré à ce jour. Enfin, les effets du système nerveux sympathique sur le métabolisme de base restent équivoques. Il a été montré qu'une faible portion de la variabilité du métabolisme de base pouvait être mise en relation avec des variations de I'activité sympathique musculaire [9]. Cependant, une activation aiguë de I'activité sympathique par l'application d'une pression négative à l'hémicorps inférieur ne modifie pas le métabolisme de base [10] alors qu'un stress mental (qui stimule l'activité sympathique et la médullosurrénale) s'accompagne d'une augmentation de $20 \%$ environ des dépenses énergétiques de base. Il est maintenant reconnu que toutes les voies qui constituent le système nerveux sympathique ne sont pas activées simultanément et il est vraisemblable que I'activation de certaines voies sympathiques soient spécifiquement associées à des effets métaboliques.

Lorsque les dépenses énergétiques sont mesurées chez un individu au repos au cours de l'administration orale ou intraveineuse de nutriments, on observe une augmentation des dépenses énergétiques appelée thermogenèse alimentaire [11]. Cette thermogenèse alimentaire est proportionnelle à la quantité de substrats administrés [12]. Elle varie selon la nature des substrats: les valeurs mesurées sont de $0 \%$ à $2 \%$ pour les lipides, de $5 \%$ à $10 \%$ pour les hydrates de carbone, et de $20 \%$ à $30 \%$ pour les acides aminés et les protéines [11]. La majeure partie de la thermogenèse alimentaire peut être expliquée par le coût énergétique associé à l'absorption et au métabolisme des aliments [13]. De plus, une part de la thermogenèse observée après administration $d^{\prime}$ hydrates de carbone est en relation avec une activation du système nerveux sympathique (thermogenèse facultative) [14].

Les dépenses de l'organisme entier augmentent lors de l'activité physique. Cette stimulation des dépenses correspond principalement à l'énergie nécessaire à la contraction musculaire. Les dépenses énergétiques mesurées croissent avec l'intensité de l'exercice. Ainsi, les dépenses énergé- tiques basales sont multipliées par un facteur de 3 à 5 lors de la marche, de 5 à 10 lors de la course à pied ou d'autres sports d'endurance. L'augmentation maximale des dépenses d'énergie dépend non seulement du type d'activité pratiquée, mais également de l'entraînement physique du sujet. En effet, la pratique répétée d'une activité physique accroît la synthèse protéique, augmente la masse musculaire et stimule également la synthèse d'enzymes oxydatives et la prolifération des mitochondries dans les groupes musculaires concernés [15]. En conséquence, la consommation maximale d'oxygène et donc les dépenses énergétiques maximales augmentent avec l'entraînement physique. L'énergie nécessaire à la contraction musculaire est fournie par I'hydrolyse des molécules d'ATP. Lors de cette hydrolyse, $40 \%$ seulement de l'énergie libérée sont convertis en travail mécanique, les $60 \%$ restants étant dissipés sous forme de chaleur. La synthèse des molécules d'ATP à partir d'ADP et de phosphate inorganique s'effectue grâce à l'oxydation intramusculaire de glucose et d'acides gras, en proportions variables selon l'intensité et la durée de l'exercice et le degré d'entraînement du sujet. Lors de l'oxydation de ces nutriments, $60 \%$ seulement de leur contenu énergétique sont transférés en énergie chimique dans la molécule d'ATP. II s'ensuit que le rendement énergétique du travail musculaire est de 0,6 (le rendement énergétique de la synthèse $d^{\prime}$ ATP) $x$ 0,4 (le rendement énergétique du transfert de l'énergie de l'ATP vers le muscle) $=0,24$ (figure 1$)$. Ce rendement énergétique de $24 \%$ ne varie pas ou peu d'un individu à l'autre.

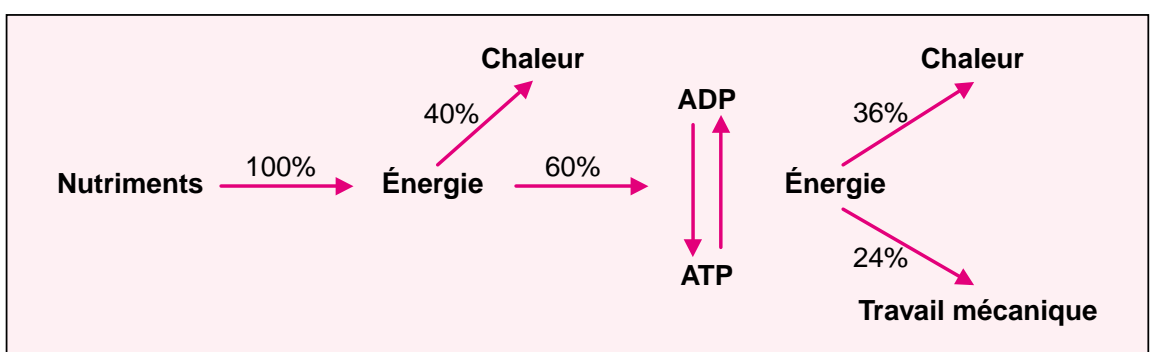

Figure 1. Rendement énergétique de la contraction musculaire. $40 \%$ de l'énergie contenue dans les aliments sont perdus sous forme de chaleur lors de la synthèse d'ATP. Lors de la contraction musculaire, $60 \%$ de l'énergie contenue dans I'ATP sont perdus sous forme de chaleur. Le rendement énergétique global de la contraction musculaire est donc d'environ $24 \%$. 


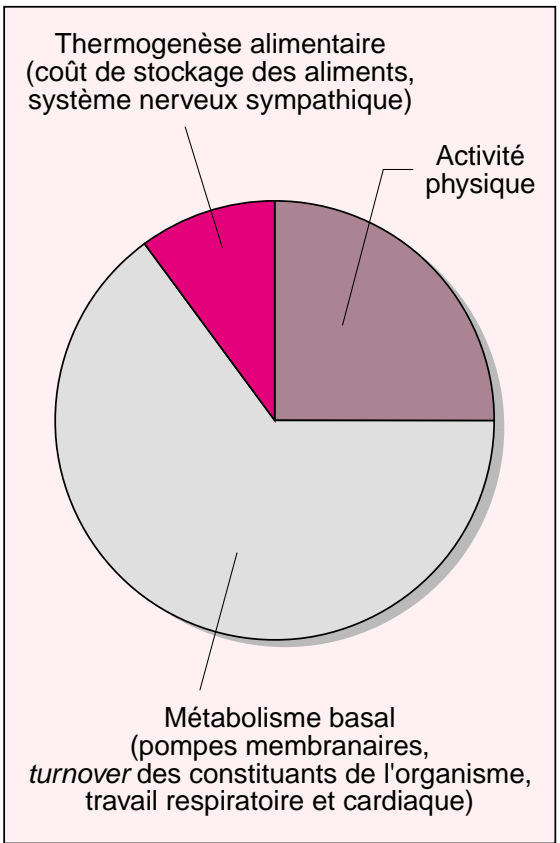

Figure 2. Composantes des dépenses énergétiques de 24 heures (individu sédentaire). La majeure partie (60$70 \%$ ) de la dépense énergétique totale est représentée par le métabolisme de base. Seuls 20-30\% des dépenses correspondent à une activité physique chez l'individu sédentaire.

peux brun [16]. Dans ce tissu, la phosphorylation oxydative peut être découplée sous l'action d'une protéine mitochondriale, I'UCP1. II s'ensuit une oxydation de substrats énergétiques associée à la production de chaleur, sans synthèse d'ATP. Le métabolisme du tissu adipeux brun joue un rôle important dans la thermogenèse induite par l'exposition au froid et la thermogenèse alimentaire chez le rongeur. Chez l'homme adulte, le tissu adipeux brun n'est pas présent en quantité importante, et son rôle est probablement moindre. Récemment, de nouvelles protéines découplantes (UCP2 et UCP3) ont été décrites dans le muscle, le tissu adipeux et d'autres types cellulaires chez l'animal et chez I'homme. Le rôle de ces protéines dans une éventuelle thermogenèse facultative reste cependant à démontrer [17].

\section{Balance énergétique et contrôle du poids corporel}

Le maintien d'un poids et d'une composition corporelle constants dépend de l'équilibre entre les apports et les dépenses d'énergie. En cas d'apport alimentaire supérieur aux dépenses d'énergie, l'excédent d'énergie consommée est stocké dans l'organisme. Il existe une hiérarchie dans l'oxydation des substrats alimentaires. L'administration d'hydrates de carbone s'accompagne de modifications neuro-endocriniennes favorisant leur utilisation immédiate. En conséquence, I'oxydation des hydrates de carbone est à moyen terme égale à leur apport et leur stockage net est nul. Il en va de même pour les protéines pour lesquelles l'oxydation journalière s'équilibre rapidement avec la quantité de protéines ingérées. En revanche, I'apport de lipides n'a pas ou peu d'influence sur leur oxydation [18]. II en résulte qu'en cas d'apport alimentaire excessif, la majorité des calories en excès est stockée sous forme de lipides dans le tissu adipeux. Il existe cependant également un accroissement de la masse non grasse de l'organisme lors de prise pondérale et il peut être estimé que chaque kilo de poids gagné correspond à environ $0,75 \mathrm{~kg}$ de tissu adipeux et à $0,25 \mathrm{~kg}$ de masse non grasse [19].

Le maintien $d^{\prime} u n$ poids corporel constant dépend d'un système de régulation complexe permettant d'adapter les apports alimentaires à des dépenses énergétiques qui peuvent être variables d'un jour à l'autre ou d'une période de la vie à l'autre. II apparaît que cette régulation ne s'opère pas au jour le jour (c'est-àdire que les apports alimentaires ne sont pas adaptés aux dépenses énergétiques du jour même), mais plutôt sur des périodes plus prolongées.

Les mécanismes responsables du maintien d'un poids corporel peuvent théoriquement faire intervenir deux mécanismes distincts. Tout d'abord, un excès de prise alimentaire peut exercer un rétrocontrôle négatif sur les prises alimentaires subséquentes. Les voies neurohormonales impliquées dans ce rétrocontrôle commencent à être élucidées, mais de nombreux points d'ombre subsistent. La prise alimentaire est réglée par I'activité de centres hypothalamiques qui reçoivent des signaux de l'ensemble de I'organisme les informant sur l'état nutritionnel prévalent. La prise alimentaire est stimulée par la sécrétion de ces centres de neuropeptides par les neurones. Le plus puissant stimulant de la prise alimentaire connu à ce jour est le neuropeptide Y (NPY) (m/s 1996, $n^{\circ} 8 / 9$, p. 974) [20], mais d'autres peptides, tels les orexines $\left(\mathrm{m} / \mathrm{s}\right.$ 1997, $n^{\circ} 10, p .1202$ et 1998, $n^{\circ} 4$, p. 498), jouent également un rôle. Une prise alimentaire exerce un rétrocontrôle négatif immédiat sur la sécrétion de NPY par I'hypothalamus par le biais d'une élévation de l'insuline [21]. A plus long terme, cependant, la prise alimentaire influe sur la composition corporelle. Plus spécifiquement, un excès alimentaire s'accompagne de modifications de l'état métabolique des cellules adipeuses, puis d'une augmentation de la masse adipeuse. Cela entraîne une stimulation d'un peptide synthétisé par les cellules adipeuses, la leptine, qui exerce un puissant effet d'inhibition de la prise alimentaire, en inhibant en partie la sécrétion de NPY hypothalamique [19].

Un excès de prise alimentaire pourrait stimuler les dépenses énergétiques de manière à empêcher un stockage inadéquat d'énergie sous forme de graisse corporelle. De tels mécanismes sont présents chez le rongeur, chez lequel la présence de tissu adipeux brun permet d'augmenter la dépense énergétique en dissipant de la chaleur lors de prise alimentaire excessive. Chez l'homme, l'existence de tels mécanismes reste spéculative. Une suralimentation ne modifie pas ou peu le métabolisme de base, la thermogenèse alimentaire ou le rendement énergétique du travail musculaire. Certains travaux, cependant, indiquent que les dépenses énergétiques globales peuvent être modifiées par la prise alimentaire. Ainsi, une suralimentation prolongée conduisant à un gain de $10 \%$ du poids initial, s'accompagne d'une augmentation des dépenses énergétiques de 24 heures supérieure à celle attendue [22]. La stimulation des dépenses énergétiques, induite par l'excès alimentaire, ne correspond pas à une augmentation du métabolisme de base ou de la thermogenèse alimentaire. Le rendement énergétique de l'exercice physique n'est pas modifié. Cette stimulation des dépenses énergétiques est donc principalement attribuable à une stimulation de la motilité non liée à l'exercice sportif (mouvements spon- 


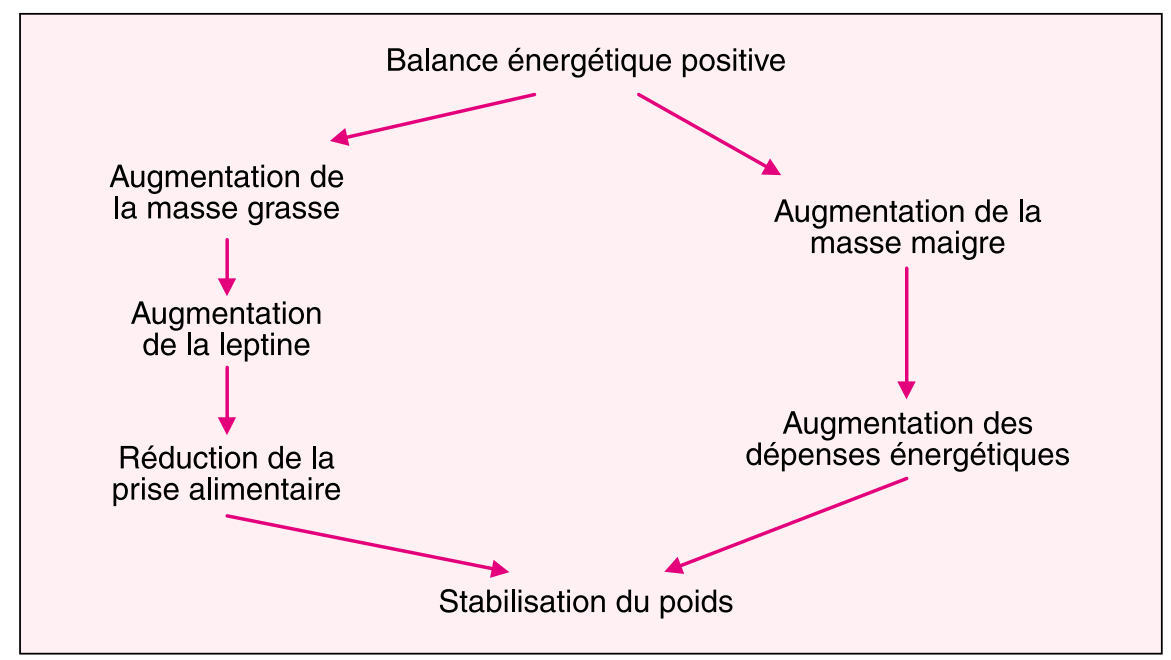

Figure 3. Mécanismes de stabilisation du poids lors d'une alimentation hypercalorique prolongée. Lors d'alimentation hypercalorique continue, le poids corporel augmente. Un gain de masse maigre entraîne une augmentation des dépenses énergétiques totales, tendant à rééquilibrer la balance énergétique. Simultanément, la masse grasse s'accroît, ce qui entraîne une sécrétion plus importante de leptine, qui tend à réduire la prise alimentaire.

tanés effectués dans le cadre des activités journalières, une part de l'activité physique dénommée fidgeting par les auteurs anglo-saxons) [23]. L'efficacité avec laquelle un apport alimentaire excessif entraîne une augmentation des dépenses énergétiques globales semble très variable selon les individus, et pourrait conditionner, dans une certaine mesure, la prise pondérale. Les mécanismes en restent inconnus.

II semble donc qu'un excès d'apport alimentaire ne modifie que peu les dépenses énergétiques exprimées par unité de masse corporelle maigre. En contre-partie, chaque kilo de poids déposé s'accompagne d'un accroissement de la masse maigre et, de ce fait, augmente les dépenses énergétiques. Il en découle que des modifications de poids et de composition corporelle constituent le mécanisme principal grâce auquel les dépenses énergétiques augmentent lors d'excès alimentaire. Un excès calorique entraîne une augmentation de poids jusqu'à ce que les dépenses énergétiques s'élèvent et qu'un nouvel état d'équilibre soit atteint [19] (figure 3).

\section{Mécanismes à l'origine de l'obésité}

La prévalence de l'obésité est en augmentation continue dans les sociétés occidentales depuis le début de ce d'activité physique semblent jouer un rôle important. Des études épidémiologiques montrent une augmentation continue du poids depuis le milieu du siècle alors que, pendant la même période, la prise alimentaire quotidienne a diminué considérablement. Cela indique que la réduction d'activité physique n'a pas été entièrement compensée par une réduction des apports alimentaires. Des données collectées chez les enfants obèses nord-américains vont dans le même sens. Une relation est observée entre le nombre d'heures passées chaque jour devant la télévision et le poids corporel [26]. De plus, une réduction du temps passé devant la télévision s'accompagne d'une augmentation de I'activité physique, d'une diminution du grignottage et $d^{\prime}$ une réduction du poids corporel.

Les mécanismes par lesquels l'exercice physique pourrait influer sur la prise alimentaire restent mal connus. Une activité physique modérée ne modifie pas la production de leptine des adipocytes à court terme [27]. II est donc concevable de supposer qu'une activité physique soutenue n'abaisse la leptine qu'à moyen terme, après qu'une réduction de la masse grasse se soit effectuée. En d'autres termes, les dépenses énergétiques liées à l'activité physique ne seront compensées par une prise alimentaire augmentée qu'après une réduction de la masse grasse de l'organisme.

\section{Conclusions}

L'activité physique ne représente qu'une part minoritaire des dépenses énergétiques globales de l'organisme chez l'homme occidental moderne. Elle semble cependant jouer un rôle fondamental dans le contrôle pondéral. Une activité physique basse favorise I'apparition d'une obésité. En cas de suralimentation, certains individus semblent stimuler leur activité physique pour prévenir une prise pondérale excessive. Les mécanismes liant le contrôle de la prise alimentaire, la balance énergétique et l'activité physique restent cependant mal élucidés. Il est possible que l'identification des mécanismes responsables du contrôle de la prise alimentaire et des dépenses énergétiques au niveau du système nerveux central ou des facteurs de contrôle des dépenses énergétiques «facultatives» ouvre des perspectives thérapeutiques nouvelles

\section{RÉFÉRENCES}

1. Schutz $Y$, Jéquier $E$. Resting energy expenditure, thermic effect of food, and total energy expenditure. In: Bray GA, Bouchard C, James WPR, eds. Handbook of obesity. New York: Marcel Dekker,1998: 443-55.

2. Jéquier $\mathrm{E}$, Felber J. Indirect calorimetry. Baillieres Clin Endocrinol Metab 1987; 1: 911-35.

3. Schoeller DA, Fjeld CR. Human energy metabolism: what have we learned from the doubly labeled water method? Annu Rev Nutr 1991; 11 : 355-73. 


\section{RÉFÉRENCES}

4. Owen OE. Resting metabolic requirements of men and women. Mayo Clinic Proc 1988; 65 : 503-10.

5. Weinsier R, Schutz Y, Bracco D. Reexamination of the relationship of resting metabolic rate to fat-free mass and to the metabolically active components of fat-free mass in humans. Am J Clin Nutr 1992; 55: 7904.

6. Randin J-P, Schutz Y, Scazziga B, Lemarchand-Béraud $T$, Felber J-P, Jéquier E. Unaltered glucose-induced thermogenesis in Graves disease. Am J Clin Nutr 1986; 43 : 738-44.

7. Webber J, Macdonald IA. Metabolic actions of catecholamines in man. Baillieres Clin Endocrinol Metab 1993; 7 : 393-413.

8. Himms-Hagen J, Ricquier D. Brown adipose tissue. In: Bray GA, Bouchard C, James WPT, eds. Handbook of obesity. New York: Marcel Dekker, 1998: 415-41.

9. Spraul M, Ravussin E, Fontvieille AM Rising R, Larson DE, Anderson EA. Reduced sympathetic nervous activity. J Clin Invest $1993 ; 92: 1730-5$.

10. Tappy L, Girardet K, Schwaller N, et al. Metabolic effects of an increase of sympathetic activity in healthy humans. Int $\int$ Obesity $1995 ; 19$ : 419-22.

11. Acheson KJ. Influence of autonomic nervous system on nutrient-induced thermogenesis in humans. Nutrition 1993; 9 : 373-80.

12. D'Alessio D, Kavle E, Mozzoli M, et al. Thermic effect of food in lean and obese men. J Clin Invest 1988; 81: 1781-9.

13. Flatt JP. The biochemistry of energy expenditure. In: Bray G, eds. Recent Advances in Obesity Research II. London: John Libbey, 1980: 211-8.

14. Acheson KJ, Jéquier E, Wahren J. Influence of $\beta$-adrenergic blockade on glucose-induced thermogenesis in man. I Clin Invest $1983 ; 72: 981-6$.

15. Physiological determinants of exercise tolerance in humans. In: Whipp BJ, Sargeant
AJ, eds. Studies in Physiology four. London: Portland Press, 1999.

16. Lowell BB, Spiegelman BM. Towards a molecular understanding of adaptive thermogenesis Nature 2000; 404: 652-60.

17. Riquier D, Bouiller F. Les protéines découplantes mitochondriales. Med $\mathrm{Sci}$ $1998 ; 14: 889-97$

18. Flatt J, Ravussin E, Acheson K, Jéquier E. Effects of dietary fat on post-prandial substrate oxidation and carbohydrate and fat balance. J Clin Invest 1985; 76: 1019-24.

19. Jéquier E, Tappy L. Regulation of body weight in humans. Physiol Rev 1999; 79: 451-80.

20. Cusin I, Rohner-Jeanrenaud F. Boucle régulatrice entre le neuropeptide $\dot{Y}$ et son altération chez le rongeur obèse. Med Sci 1998 ; 14 : 907-13.

21. Schwartz MW, Dallman MF, Woods SC. Hypothalamic response to starvation: implications for the study of wasting disorders. Am J Physiol 1995; 269: R949-57.

22. Leibel RL, Rosenbaum M, Hirsch J. Changes in energy expenditure resulting from altered body weight. $N$ Engl J Med $1995 ; 332: 621-8$

23. Levine JA, Eberhardt NL, Jensen MD. Role of nonexercise activity thermogenesis in resistance to fat gain in humans. Science 1999; $283: 212-4$

24. Oppert JM, Rolland-Cachera MF. Prévalence, évolution dans le temps et conséquences économiques de l'obésité. Med Sci $1998 ; 14: 939-43$.

25. Perusse L, Bouchard C. Genotype-environment interaction in human obesity. Nutr Rev 1999; 57: S31-7.

26. Robinson TN. Reducing children's television viewing to prevent obesity: a randomized controlled trial. JAMA 1999; 282: mized $1561-7$

27. Dirlewanger $M$, Di Vetta $V$, Giusti $V$, Schneiter $P$, Jéquier $E$, Tappy L. Effect of moderate physical activity on plasma leptin concentration in humans. Eur Appl Physiol $1999 ; 79: 331-5$ $\mathbf{m} / \mathbf{S} 2000$

\section{Summary}

Energy expenditure, body composition and physical activity in humans

The energy expenditure of man can be subdivided into the basal metabolic rate, the dietary thermogenesis (or thermic effect of food) and the energy expanded in physical activity. The latter corresponds to the energy expanded to support muscle contraction. The energetic efficiency of skeletal muscle work is about $25 \%$. In sedentary individuals, the energy expanded in physical activity represents a relatively minor portion $(20-30 \%)$ of daily energy expenditure. Obesity results from an imbalance between energy intake and expenditure, resulting in positive energy balance during the period of weight gain. At the phase of obesity maintenance, an even energy balance is restaured through an increase in body weight and lean body mass. During overfeeding there is little adaptative changes in energy expenditure. Overfeeding has no marked effect on basal metabolic rates or on dietary thermogenesis. Recent observations however suggest that stimulation of physical activity may occur in some individuals during overfeeding, and may contribute to prevent or limit weight gain.

\section{TIRÉS À PART}

L. Tappy.

\section{Prix Nestlé 2001 "L'homme et sa nutrition"}

Ce prix récompense une équipe française pour un ensemble de travaux. Il est destiné à promouvoir la recherche dans les domaines de la nutrition humaine, des technologies alimentaires et des risques associés tout au long de la filière agro-alimentaire, en fonction de leurs conséquences sur la santé. 